\title{
Design of Selectable Modems for MC-CDMA Based on Software Defined Radio
}

\author{
Ali Kareem Nahar ${ }^{\mathrm{a}, \mathrm{b}}$ \\ ${ }^{a}$ Faculty of Electrical and Electronic \\ Engineering, University Malaysia Pahang , \\ 26600Pekan, Pahang, Malaysia
}

MC-CDMA technique is the combination of Orthogonal Frequency Division Multiplexing (OFDM) technique and Code Division Multiple Access (CDMA) technique and collects the benefits of both techniques to provide higher data rates and greater flexibility for voice, data, video and internet services for future wireless systems. In this paper MC-CDMA system based on Software Defined Radio (SDR) was proposed. The proposed data spread model consists of gold code and Selectable six modulation types (BPSK, QPSK, 8QAM, 16QAM, 32QAM and 64QAM). In addition, OFDM is designed by both FFT and IFFT for detecting ideal channel. The programming is done by using MATLABSimulink tool as well as M-files presented for each modem. Matlab 13A. The transmitter send 4, 3 and 2 bit to the receiver in which the system indicate is too big for 4 and 3 bit therefore the transmitted but reduced to two bit for successfully system work. To achieve optimum encoding and decoding signal the all modulation techniques use $5 \mathrm{MHz}$ to $20 \mathrm{MHz}$ spectrum frequency. Moreover the bandpass signal generation has optimal utilized area to satisfy the required sampling rate

\section{INTRODUCTION}

Recently, the growth of video, voice and data communication, the users demanded high date rate over the Internet wireless environment where the spectral resource is scarce. To fulfill the requirements SDR-CDMA is very efficient way to overcome inter-symbol interference (ISI) on frequency selective channels [1].

Many research focus on OFDM scheme which has severed disadvantages such as nonlinear amplification, sensitivity to frequency offset and difficulty in subcarrier synchronization [2]. MCCDMA is a combination of CDMA and OFDM and has the benefits of both systems $[4,5]$. Thus, the parameters of OFDM become the basic parameters of MC-CDMA. In $[3,4]$ proposed OFDM based on wavelet, where both FFT and IFFT blocks are replaced by an inverse discrete wavelet transform (IDWT) and discrete wavelet transform (DWT)respectively. In [5,6] propose MC-CDMA system based on a combination of OFDM and CDMA system for better robustness against multipath, interference rejection, and impulse noise frequency reuse, etc. In [7] proposed OFDM for broad-band local area wireless based on standards IEEE802.11a $[8,9]$.

\author{
Yusnita Rahayu ${ }^{a}$ \\ $b$ Universities of Technology, Department of \\ Electrical Engineering, Baghdad, Iraq
}

In this paper focus inn analyze the parameters of OFDM selectable modulation in MC-CDMA. The simulation parameters considered are: guard time interval, sampling rate, symbol duration, and number of data subcarriers. The analysis carried out using MATLAB. The OFDM and MC-CDMA analyze under different parameters to determine the better of the two for the modern wireless communications.

\section{RELATED WORK}

In the recent past, a number of study projects in the field of SDR networking have been presented. In [10] proposed a new design of CDMA digital transmitter for a multi-standard SDR base band stage. The platform involves of reconfigurable and reprogrammable hardware platform which provide different standards with a common platform, and implemented with FPGA by create VHDL model of CDMA transmitter. In [11] introduce a basic acquisition system for finding and classifying Base Stations (BSs) in visibility in the framework of a CDMA wireless positioning system, based on IS95 cellular standard. In [12], concentration on the importance of MC-CDMA and use adaptive modulation, the exploitation of fluctuations channel quality, so that they can exchange more traffic multimedia using the same bandwidth, a high efficiency in bandwidth and diversity inherent to the channel fading as compared with OFDM and DS-CDMA in the Fig. 1 shown

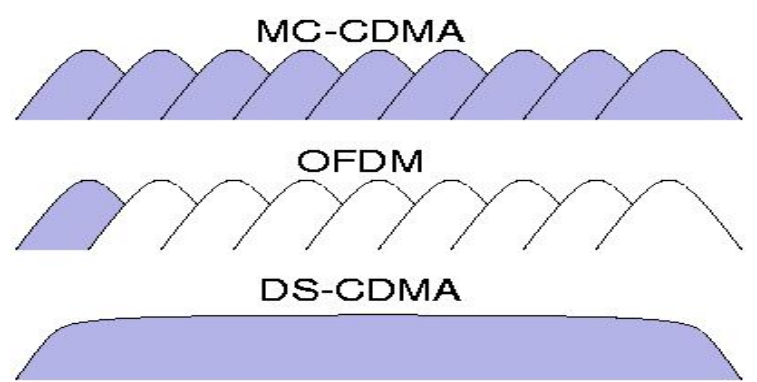

Fig. 1 MC-CDMA and DS-CDMA use the whole bandwidth

Each of these blocks was tested using FPGA advantages 7.2 software during design process; the same process was done at the receiver part where using each of the modules was experienced during design process [13]. Moreover, Mahbub, [14] 
proposed an implementation of DS-CDMA transmitter. In [15] was a show implementation topic of a digital transmitter for an OFDM through adjusted VHDL in contradiction of system generator results. Canet's work is absorbed on solutions for the OFDM signal generation in IF and base-band. Implementation of SDR implies more specific design and analysis procedures than the implementation of conventional transceiver systems. Selection of hardware components for transceiver implementation, that follows the SDR concept, is the first and crucial step necessary for its implementation. All selected hardware components together form a hardware platform for SDR creation. During the process of forming a hardware platform it is necessary to achieve a compromise between desired, scalability, flexibility, modularity and performance of the SDR system [16]. Scalability is related to modularity, and it allows the system to be enhanced to improve capability such as increasing number of channels that a base station could handle. In addition, flexibility is the capability of a system to switch variety of air-interfaces and protocols, even if they have yet to be defined. Also, modularity of a system allows easy replacement or progress of subsystems to take advantage of new technologies [16]. In [17], that they discussed the M-QAM for forward link of MC-CDMA schemes with interference dissolution to support high data rate service, and provided an analytical BER performance of the system. In [18], emphasizes the suitability of high level design tools when designing sophisticated systems, and the importance to design FPGA systems rather than ASIC for accomplishing one day the SDR idea and give a high level overview of the FPGA implementation, that work emphasizes the packet detection, synchronization, preamble correlator, channel estimation and equalization; that is primarily at the OFDM receiver for the
IEEE802.11. In [19], developed a SDR networking is platform using GNU Radio and the USRP. They integrated a Tun/Tap device into their solution and additionally studied the impact of channel quality and different modulation schemes. In [20], based on their previous observations, MacKenzie et al. developed a split functionality approach in order to overcome the communication delays introduced through SDR and the USRP. Moving time sensitive functionality closer to the radio promises better performance in terms of delay. The drawback, however, is the decreased flexibility and higher implementation complexity.

\section{THE PROPOSED SYSTEM}

The general layout for proposed system is shown in Fig. 2. The main parts and functions of the implemented proposed system are:

1. Transmitter: The transmitter is responsible for generating the symbols of the transmitted data which is transmitted over a wireless channel. Six modems are used in this transmitter, these are BPSK, QPSK, 8QAM, 16QAM, 32QAM and 64QAM that can be select which type of these modems above is turned on and the others are turned off by the response of the selectable modem unit.

2. Receiver: This is responsible for data reception and demodulation of the received data. The selectable modem unit is used in the receiver section to decide which demodulation and decision circuit are used to demodulate the received modulated signal and received the data signal.

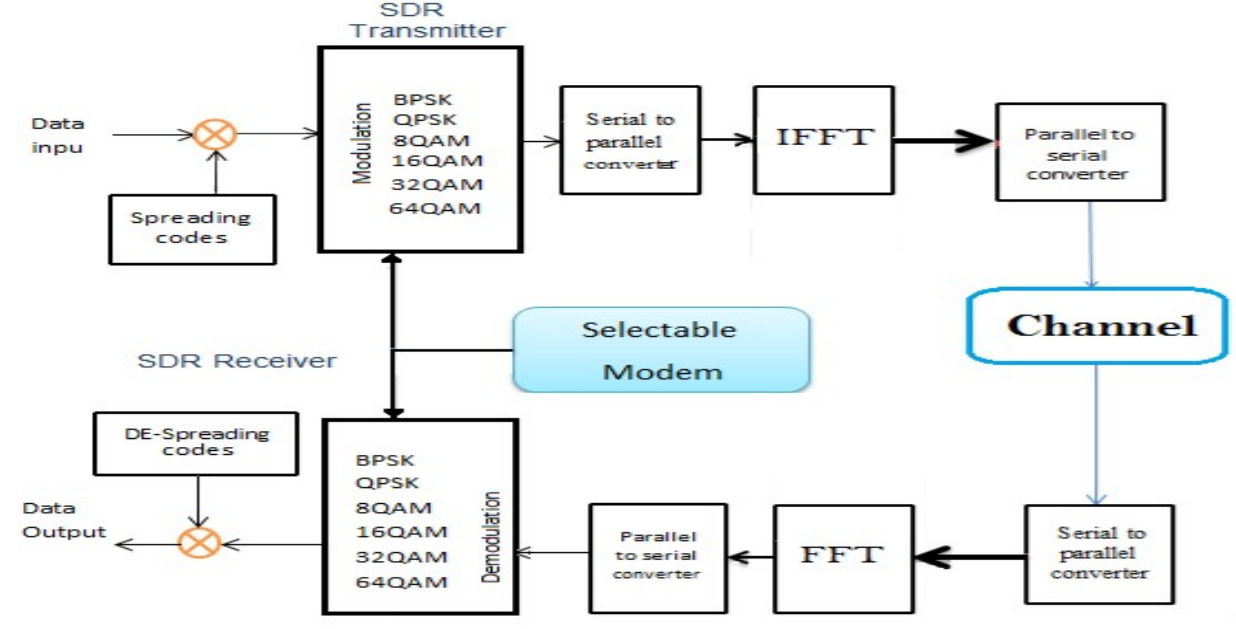

Fig.2. Proposed system layout 
Fig. 3 describes the design and implementation procedure used for the proposed SDR system. The SDR parameters are set up according to IEEE802.16e CDMA standard. Then, the design is implemented as a model using MATLAB

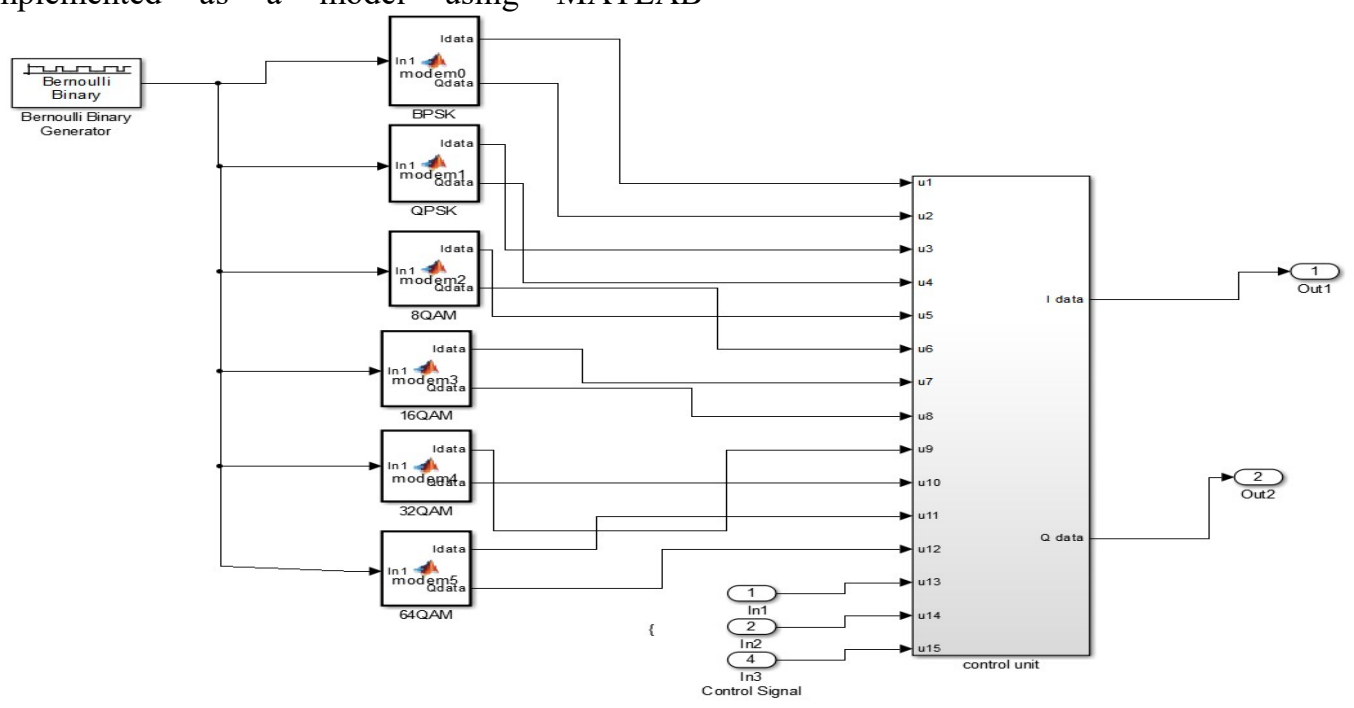

Fig. 3 The proposed SDR system implemented in MATLAB

\section{RESULT AND DISCUSSION}

The system parameters setting includes specifying the different types of modulation/demodulation and other related system operations that the SDR could handle [21]. Table 1. shows the proposed design system parameters. The SDR system is very flexible and can change its parameters easily.

The variation of the BER are performed according to the variation ratio for energy of data bit to the power spectrum density $\left(\mathrm{E}_{\mathrm{b}} / \mathrm{N}_{\mathrm{o}}\right)$. Fig. 4 shows the performance of modulation over channel. Table 2 . shows the representation of data which is greatly generated. Fig. 5 represents I and Q-symbol which is multiplied by PN-I and PN-Q respectively. Fig. 6 represents the I and Q signals with 64-QAM modulation transmitted in MC-CDMA. (combination MATLAB-Simulink and M-file) and functional simulation is performed to performance evaluation.

Table. 1: Design system parameters

\begin{tabular}{|l|l|l|}
\hline paramter & Selected types or values & explain \\
\hline Modlation type & $\begin{array}{l}\text { BASK,QPSK,8QAM,16Q } \\
\text { AM,32QAM,64QAM }\end{array}$ & $\begin{array}{l}\text { BPSK, QASK and M-QAM } \\
\text { used in this system to increase } \\
\text { data rate of transmitssion }\end{array}$ \\
\hline IF frequency & $5-20 \mathrm{MHz}$ & $\begin{array}{l}\text { Moderate frequency can be used } \\
\text { to implement SDR system }\end{array}$ \\
\hline Sampling frequency & $100 \mathrm{MHz}$ & $\begin{array}{l}\text { This value is selected for better } \\
\text { simulation results }\end{array}$ \\
\hline FFT size & 256 & $\begin{array}{l}\text { 8-inputs and the values of the } \\
\text { twiddle factor, each equation } \\
\text { as paths even and odd }\end{array}$ \\
\hline Sprading cods & Gold code & $1.2288 \mathrm{Mp} / \mathrm{s}$ \\
\hline
\end{tabular}




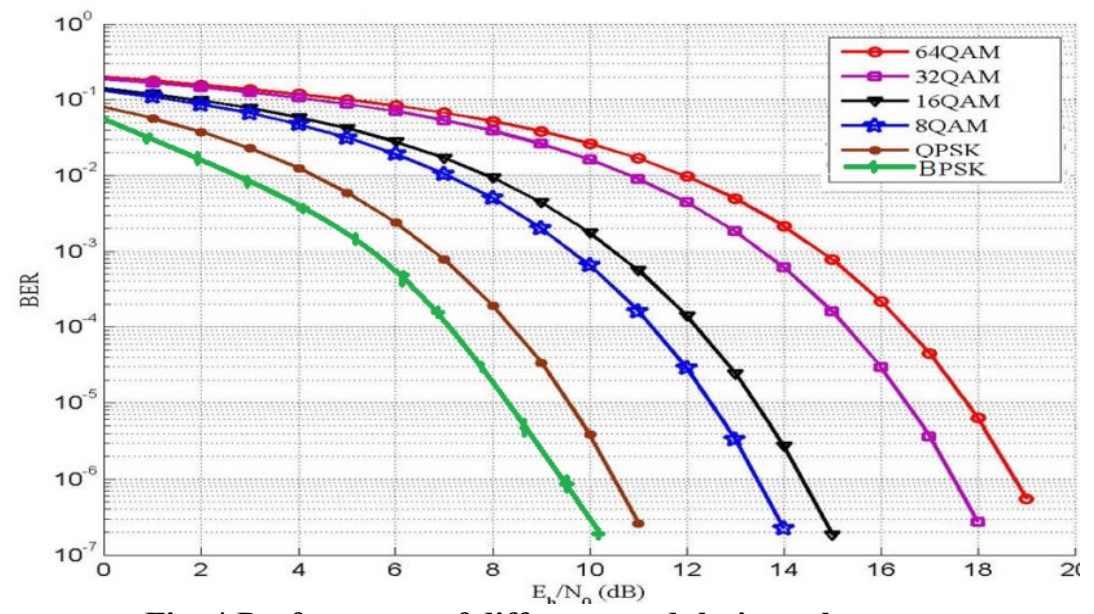

Fig. 4 Performance of different modulation schemes

Table. 2 Representation of 32-QAM, 16-QAM, 8QAM, QPSK andBPSK signals

\begin{tabular}{|c|c|c|c|c|c|c|c|c|c|c|}
\hline \multirow[b]{2}{*}{$\begin{array}{l}\text { symbo } \\
1\end{array}$} & \multicolumn{2}{|l|}{ 32QAM } & \multicolumn{2}{|c|}{ 16QAM } & \multicolumn{2}{|l|}{ 8QAM } & \multicolumn{2}{|l|}{ QPSK } & \multicolumn{2}{|l|}{ BPSK } \\
\hline & $\begin{array}{l}\text { I- } \\
\text { Channe } \\
1\end{array}$ & $\begin{array}{l}\text { Q- } \\
\text { Channe } \\
1\end{array}$ & $\begin{array}{l}\text { I- } \\
\text { Channe } \\
1\end{array}$ & $\begin{array}{l}\text { Q- } \\
\text { Channe } \\
1\end{array}$ & $\begin{array}{l}\text { I- } \\
\text { Channe } \\
1\end{array}$ & $\begin{array}{l}\text { Q- } \\
\text { Channe } \\
1\end{array}$ & $\begin{array}{l}\text { I- } \\
\text { Channe } \\
1\end{array}$ & $\begin{array}{l}\text { Q- } \\
\text { Channe } \\
1\end{array}$ & $\begin{array}{l}\text { I- } \\
\text { Channe } \\
1\end{array}$ & $\begin{array}{l}\text { Q- } \\
\text { Channe } \\
1\end{array}$ \\
\hline 0 & -3 & 5 & -3 & 3 & -3 & 1 & 1 & 1 & 1 & 0 \\
\hline 1 & -1 & 5 & -3 & 1 & -3 & -1 & -1 & 1 & -1 & 0 \\
\hline 2 & -1 & -5 & -3 & -1 & -1 & 1 & -1 & -1 & & \\
\hline 3 & -3 & 5 & -3 & -3 & -1 & -1 & 1 & -1 & & \\
\hline 4 & -5 & 3 & -1 & 3 & 1 & 1 & & & & \\
\hline 5 & -5 & 1 & -1 & 1 & 1 & -1 & & & & \\
\hline 6 & -5 & -1 & -1 & -1 & 3 & 1 & & & & \\
\hline 7 & -5 & -3 & -1 & -3 & 3 & -1 & & & & \\
\hline 8 & -3 & 3 & 1 & 3 & & & & & & \\
\hline 9 & -3 & 1 & 1 & 1 & & & & & & \\
\hline 10 & -3 & -1 & 1 & -1 & & & & & & \\
\hline 11 & -3 & -3 & 1 & -3 & & & & & & \\
\hline 12 & -1 & 3 & 3 & 3 & & & & & & \\
\hline 13 & -1 & 1 & 3 & 1 & & & & & & \\
\hline 14 & -1 & -1 & 3 & -1 & & & & & & \\
\hline 15 & -1 & -3 & 3 & -3 & & & & & & \\
\hline 16 & 1 & 3 & & & & & & & & \\
\hline 17 & 1 & 1 & & & & & & & & \\
\hline 18 & 1 & -1 & & & & & & & & \\
\hline 19 & 1 & -3 & & & & & & & & \\
\hline 20 & 3 & 3 & & & & & & & & \\
\hline 21 & 3 & 1 & & & & & & & & \\
\hline 22 & 3 & -1 & & & & & & & & \\
\hline
\end{tabular}




\begin{tabular}{|l|l|l|}
\hline 23 & 3 & -3 \\
\hline 24 & 5 & 3 \\
\hline 25 & 5 & 1 \\
\hline 26 & 5 & -1 \\
\hline 27 & 5 & -3 \\
\hline 28 & 3 & 5 \\
\hline 29 & 1 & 5 \\
\hline 30 & 1 & -5 \\
\hline 31 & 3 & -5 \\
\hline
\end{tabular}
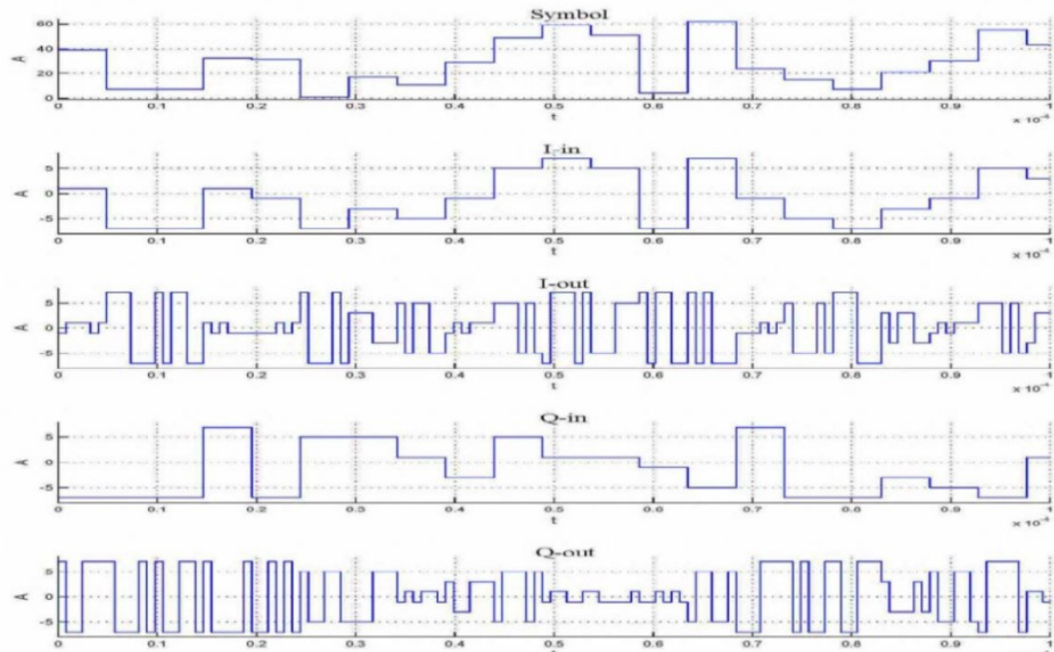

Fig. 5 I and Q-symbol multiplied by PN-I and PN-Q with 64QAM

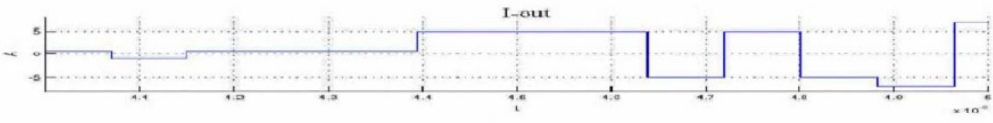

T-cot + conswo

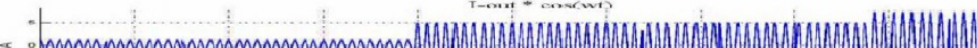
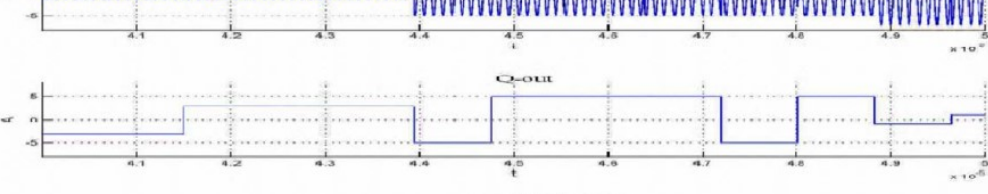

D-out $-\sin (w t)$

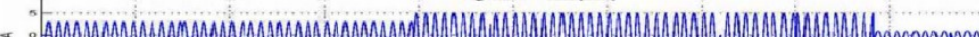

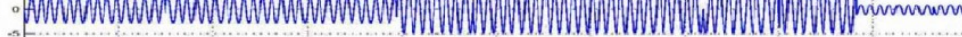

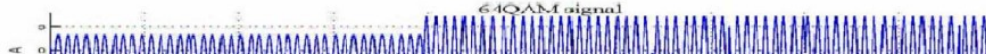

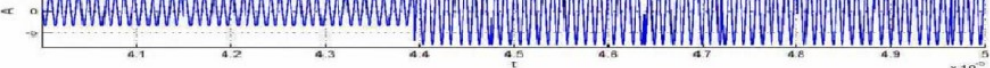

Fig. 6 MC-CDMA using 64-QAM transmitted signal. 


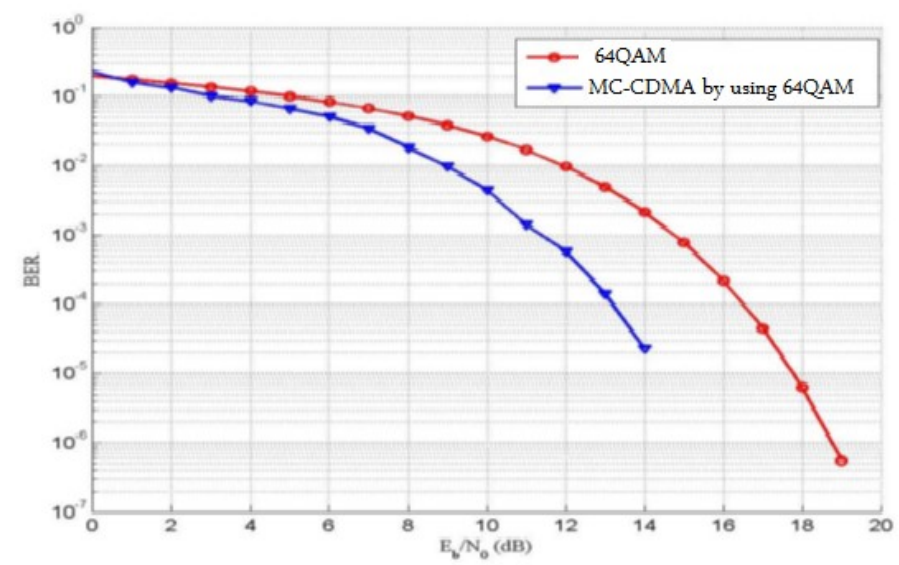

Fig. 7 Simulation results of MC-CDMA by using 64-QAM modulation

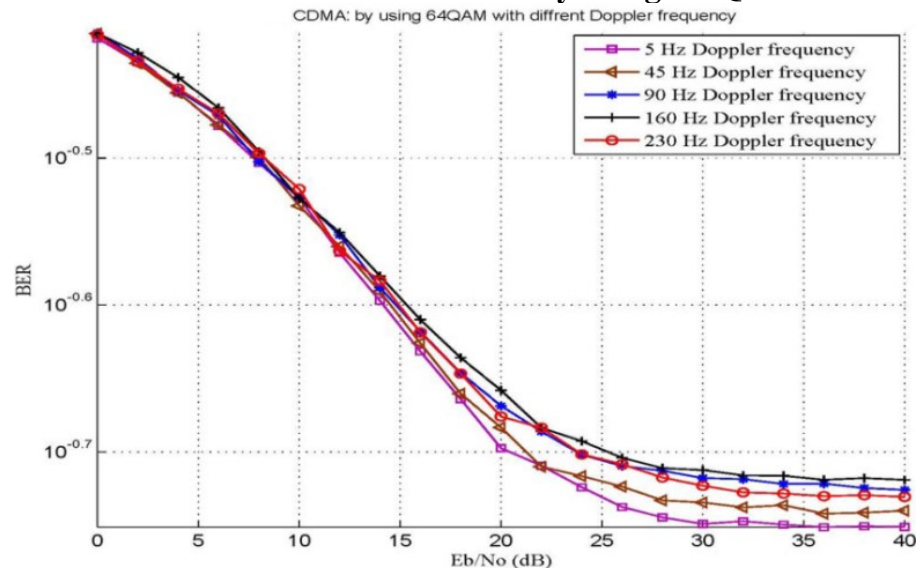

Fig. 8 Simulation results of MC-CDMA by using 64-QAM modulation

\section{CONCLUSIONS}

In this paper, selectable six models were proposed to enhance the performance of OFDM scheme. Performance of proposed MC-CDMA systems enhanced with increasing processing gain, but with large processing gain the performane of systems degraded. Multimode soft decision circuit to determine the regions of the received signal acceptable to define the final output data. The decision circuit includes 8, 16, 32 and 64 regions. Division of input data by the variable factor according to number of bit per symbol. The variable factor is 2,3,4,5 and 6 and is determined by selectable circuits. Generation of bandpass signal for six modems in order to set the IF signal required by SDR systems, as well as the generation of the bandpass signal which has optimal utilized area with satisfied the required sampling rate. SDR will have a key role to play, in the cognitive systems. We have suggested the SDR algorithms for successful data transmission in bandwidth obtainable. The performance of proposed MCCDMA schemes enhanced through increasing processing gain, but with large processing gain the performance of systems degraded.

\section{References}

1. Kumar SS, Sukanesh R. Performance Analysis of Multi-Carrier Code Division Multiple Access System Under Clipping Noise. European Journal of Scientific Research 2009; 38(4):590-595.

2. Sklar B. Digital Communications Fundamentals and Applications. Prentice Hall, 2nd Edition, 2001.

3. Vasuk H. Orthogonal Frequency Division Multiplexing. ESE505 Course, Electrical Eng. Department, State University of New York, November 1999.

4. Zhang H, yuan D. Research of DFTOFDM and DWT-OFDM on Different Transmission Scenarios. ICITA Proceeding, 2004; 28(5): 31-33

5. Nee R, Prasad R. OFDM for Wireless Multimedia Communication. Artech House, London, 2000.

6. Lawrey E. Adaptive Techniques for Multiuser OFDM. Ph.D. Thesis, James Cook University, December 2001.

7. Van Nee RDJ et al. New high-rate wireless LAN standards. IEEE Commun. Mag. 1999; 37(12):82-88. 
8. Technical S. Broadband Radio Access Networks (BRAN); HIPERLAN Type 2; Physical (PHY) layer. ETSI TS 2002; V $\{1.2 .2\} 101-475$.

9. IEEE 802.11ad. Wireless LAN Medium Access Control (MAC) and Physical Layer (PHY) specifications. 2012, IEEE Standard for Information technology

10. Mohamed $\mathrm{K}$ et al. Implementation of CDMA Transmitter for a Multi-standard SDR Base Band Platform. Asia-Pacific Conference on communications, IEEE, 2007; E-ISBN 978-1-4244-1374-4:303306.

11. Angelis GD, Baruffa G, Cacopardi S. Parallel PN Code Acquisition for Wireless Positioning in CDMA Handsets. 5th Advanced Satellite Multimedia Systems Conference and the 11th Signal Processing for Space Communications Workshop, IEEE, 2010; 343-348.

12. Grewal V, Sharma K. Performance Evaluation of Wi-MAX Network with AMC and MCCDMA for Mobile Environments. International Journal of Multimedia and Ubiquitous Engineering October, 2012; 7 (4): 107-118.

13. Mohamed MA et al. A Novel implementation of OFDM using FPGA. International Journal of Computer Science and Network Security 2011; 11 (11): 4348.

14. Mahbub TS, Ahmed S, Rokon IR. Transmitter Implementation Using DSCDMA Technique in FPGA Using Verilog HDL. International Conference on Electrical, Electronics and Civil Engineering, 2011.

15. Dash $\mathrm{T}$ K. Realization of Optimized OFDM System using FPGA on Altera Quartus3.0. International Journal of Engineering Research \& Technology (IJERT) November, 2013; 2 (11): 962968.

16. Reed JH. Software Radio: A Modern Approach to Radio Engineering. Prentice Hall Professional, 2002.

17. Xia B, Wang J. Analytical Study of QAM with Interference Cancellation for HighSpeed Multi-code CDMA. IEEE Transactions on Vehicular Technology, 2005; 549(3): 1070-1080.

18. Mohamed MA et al. Implementation of the OFDM Physical Layer Using FPGA. IJCSI International Journal of Computer Science Issues March 2012; 9(2): 612618.

19. Li X A et al. Case Study of A MIMO SDR Implementation. In Proceedings of IEEE MILCOM, 2008; E-ISBN: 978-1-42442677-5: 1-7.

20. McGettrick $S$ et al. A Split MAC Approach for SDR Platforms. IEEE Transactions on Computers 2014; 1(99):1.

21. Bouacha A, Bendimrad FT. Performance Study of the MC-CDMA as Physical Layer for Mobile Wi-Max Technology. International Journal of Computer Networking, Wireless and Mobile Communications (IJCNWMC) 2013; 3(2): 47-52 